\title{
Frecuencia y características epidemiológicas de las Ente- robacterias productoras de betalactamasas de espectro extendido en la unidad de cuidados intensivos de un hospital del norte del Perú
}

\author{
Lesvia Esperanza Pacherres-Bustamantez 1,a, Franklin Rómulo Aguilar-Gamboa 2,b, Heber Silva-Díaz ${ }^{3, c}$
}

\section{RESUMEN}

Objetivos: Describir la frecuencia y características epidemiológicas de las enterobacterias productoras de betalactamasas de espectro extendido (BLEE), aisladas desde pacientes de la unidad de cuidados intensivos (UCl) del Hospital Regional Docente las Mercedes, Chiclayo, durante enero a abril del 2018. Material y métodos. Estudio observacional descriptivo de temporalidad retrospectiva. La población de estudio estuvo constituida por 134 pacientes. La recolección de los datos se realizó mediante análisis documental del registro de microbiología del laboratorio clínico del mismo hospital. Resultados. La población de estudio tuvo una mediana de 30 años de edad (RIC: 31 a 72 ); de los cuales 40,3\% fueron positivos al cultivo microbiológico, de estos, el $51,9 \%$ de enterobacterias produjeron BLEE: Escherichia coli $(64,29 \%)$ y Klebsiella pneumoniae $(35,71 \%)$. Estas bacterias presentaron sensibilidad a los carbapenémicos $(96,4 \%)$ y resistencia al ciprofloxacino $(78,6 \%)$. Conclusiones. Las enterobacterias productoras de BLEE son frecuentes en la población estudiada y presentan alta resistencia al ciprofloxacino, los que revelan un actual problema de salud pública en la región.

Palabras clave: Resistencia betalactámica, Cuidados Críticos, Farmacorresistencia Microbiana (Fuente: DeCS-BIREME).

\section{Frequency and epidemiological characteristics of the ex- tended-spectrum beta-lactamase-producing enterobacte- ria in the intensive care unit of a hospital in northern Peru}

\section{ABSTRACT}

Objectives: To describe the frequency and epidemiological characteristics of the extended-spectrum beta-lactamase-producing enterobacteria (ESBL) isolated from the intensive care unit (ICU) patients of the Hospital Regional Docente Las Mercedes de Chiclayo, from January to April 2018. Material and methods. Observational study of retrospective temporality. The population consisted of 134 patients. The data collection was carried out through a documentary analysis of the microbiology registry of the clinical laboratory of the same hospital. Results. The study population had a median of 30 years of age (RIC: 31 to 72 ); of which $40.3 \%$ were positive to the microbiological culture, of these, $51.9 \%$ of enterobacteria produced ESBL: Escherichia coli (64.29\%) and Klebsiella pneumoniae (35.71\%). These bacteria showed sensitivity to carbapenems (96.4\%) and resistance to ciprofloxacin (78.6\%). Conclusions. The ESBL-producing bacteria are frequent in the studied population and present high resistance to ciprofloxacin, which reveal a current public health problem in the region.

Key words: beta-lactam resistance, Critical Care, Antimicrobial Drug Resistance (Source: MeSH-NLM).

\footnotetext{
${ }^{1}$ Facultad de Ciencias de la Salud y Medicina Humana, Universidad Alas Peruanas, Lambayeque, Perú..

${ }^{2}$ Laboratorio de Inmuno-Virología, Hospital Regional Lambayeque, Lambayeque, Perú.

${ }^{3}$ Dirección de Investigación, Hospital Regional Lambayeque, Lambayeque, Perú.

a Tecnólogo Médico.

b Biólogo Microbiólogo, especialista en microbiología clínica.

c Biólogo, maestro en ciencias.
} 


\section{INTRODUCCIÓN}

Las Betalactamasas de espectro extendido (BLEE) son enzimas mediadas por plásmidos, producidas por bacilos gramnegativos (enterobacteriacea) como Escherichia coli, Klebsiella pneumoniae y Proteus mirabilis. Sin embargo, también se ha registrado su presencia en bacterias: Proteus vulgaris, Serratia spp., Salmonella spp., Pseudomonas aeruginosa y Acinetobacter spp. ${ }^{(1,2)}$. Estas enzimas confieren resistencia a penicilinas, cefalosporinas de primera a la cuarta generación y aztreonam, lo que condiciona el uso de carbapenemes y otras drogas de amplio espectro. Este mecanismo de resistencia es muy frecuente tanto en el ámbito comunitario como hospitalario; en este último, principalmente en la unidad de cuidados intensivos (UCI), neonatología y emergencia, donde la estancia prolongada, métodos invasivos y terapia de amplio espectro hacen susceptible al paciente de ser colonizado por este tipo de bacterias. Por ello, representan un problema de salud pública relevante que se ha extendido rápidamente en diferentes países del mundo ${ }^{(3-5)}$.

La Organización Mundial de la Salud (OMS) informa que la resistencia a los antimicrobianos constituye una amenaza creciente para la salud pública mundial que requiere la adopción de medidas por parte de todos los sectores gubernamentales y de la sociedad en general. La aparición de cepas resistentes es un fenómeno natural que ocurre cuando los microorganismos se reproducen de forma errónea o se intercambian características de resistencia, pero el uso inadecuado de antimicrobianos también acelera su aparición ${ }^{(6)}$.

El estudio de la sensibilidad a antimicrobianos en bacterias productoras de BLEE representa un tema muy importante en la lucha frente a este fenotipo de resistencia bacteriana. Existen muchos estudios que buscan nuevas drogas y principios activos para hacer frente a este grave problema de salud pública. $Y$ es que, aunque se conoce que los carbapenemes son el tratamiento de elección para estos fenotipos de resistencia, se evita su uso debido a que se relacionan directamente con la aparición de bacterias productoras de carbapenemasas, aún más nocivos. En este sentido, antibióticos como fosfomicina, trometamol, temocilina, ceftazidima/avibactam y ceftolozano/tazobactam están siendo evaluados como posibles tratamientos que eviten o reduzcan el uso de carbapenemes ${ }^{(7,8)}$.

En el Perú el 2017 se reportó una mortalidad atribuible a bacteriemias por enterobacterias de $41,2 \%$, de las cuales $54,3 \%$ pertenecieron al grupo bacterias productoras de BLEE ${ }^{(9)}$. Así mismo, otros estudios describieron a $E$. coli $y$ Klebsiella spp. productoras de BLEE como factor asociado a mortalidad temprana en bacteriemia, y que además desarroIlaron mayores complicaciones. Las principales causas para estos hechos ha sido el uso excesivo de cefalosporinas de amplio espectro y la falta de control en el uso de antibióticos (10).

En la región Lambayeque, se han reportado una elevada frecuencia de infecciones producidas por enterobacterias productoras de BLEE, de la cual principalmente E. coli es causante y cuya tasa puede alcanzar el $31 \%$ en infecciones de origen urinario ${ }^{(11,12)}$.

Según los registros en el área de microbiología del Hospital Regional Docente "Las Mercedes" de Chiclayo (HRDLM), actualmente se reporta alta frecuencia de aislamientos de bacterias resistentes a los antimicrobianos, donde la producción de BLEE es la causa más importante de resistencia en los últimos años. Si bien los aislamientos de bacterias resistentes preceden de todos los servicios del hospital, es en la $\mathrm{UCl}$ donde se observa la mayor frecuencia de resistencia a múltiples antimicrobianos.

Por esta razón el presente estudio describió la frecuencia y características epidemiológicas de las enterobacterias productoras de BLEE, aisladas desde pacientes de la UCI del HRDLM, durante enero a abril del 2018.

\section{MATERIAL Y MÉTODOS}

\section{Tipo y diseño de investigación}

Estudio observacional descriptivo de temporalidad retrospectiva.

\section{Población y muestra}

La población estuvo constituida por 134 pacientes procedentes de la UCl del HRDLM de Chiclayo, entre enero a abril del 2018. El estudio fue censal en la población. Se excluyeron aquellos registros con datos incompletos o ilegibles.

\section{Técnicas e instrumentos de recolección de datos}

La recolección de los datos de las variables en estudio se realizó mediante la técnica del análisis documental, usando como fuente el registro de microbiología del laboratorio clínico del hospital. Los datos se registraron en una ficha de recolección de datos tomando en cuenta todos los aislamientos confirmados para la producción de BLEE realizados mediante el método de aproximación de discos (Método francés), el cual se realizó empleando un disco de amoxicilina/ácido clavulánico ubicado a $20 \mathrm{~mm}$ de cefalosporinas de tercera generación y aztreonam.

\section{Consideraciones éticas}

Para la realización del estudio se contó con la autorización institucional del HRDLM. Se garantizó en todo momento el cumplimiento de los criterios de autonomía y anonimato de cada participante mediante el acceso restringido de los investigadores los datos y registros.

\section{Análisis estadístico}

Las mediciones sobre las variables de estudio, fueron inicialmente tabuladas y ordenadas en una hoja de Microsoft Excel 2013. El análisis de las variables cualitativas se rea- 
lizó mediante el cálculo de frecuencias absolutas y relativas (porcentajes), e intervalos de confianza al 95\% (IC95\%); y las variables cuantitativas, mediante medidas de tendencia central y dispersión, previa determinación de la normalidad con la prueba de Shapiro-Wilks. Los análisis se realizaron usando los programas informáticos Microsoft Excel 2013, infostat/E versión 8 y SPSS versión 22.0.

\section{RESULTADOS:}

Se estudió 134 pacientes registrados en el laboratorio de microbiología y atendidos en la UCI del HRDLM, durante enero hasta abril del 2018. La mediana de edad fue de 60 años, rango intercuartílico de 31 a 72 años. Asimismo, la población en estudio se caracterizó porque el 50,8\% tuvo más de 60 años. La mitad de los pacientes fueron de sexo masculino $(55,2 \%)$; mientras que el tipo de muestra más frecuente fue la secreción bronquial (55,9\%). Cabe señalar que el $40,3 \%$ de la población estudiada registró cultivo positivo (IC95\%: 32,0 a 48,6) (Tabla 1).

Tabla 1. Características de los pacientes de la UCl del HRDLM $(n=134)$, Enero a abril del 2018

\begin{tabular}{lcc}
\hline \multicolumn{1}{c}{ Variables } & N & $(\%)$ \\
\hline Edad & 55 & $(41,0)$ \\
18 a 59 & 68 & $(50,8)$ \\
60 a más & 11 & $(8,2)$ \\
Menos de 18 & & \\
Sexo & 60 & $(44,8)$ \\
Femenino & 74 & $(55,2)$ \\
Masculino & & \\
Muestra & 75 & $(55,9)$ \\
Sec. Bronquial & 25 & $(18,7)$ \\
Orina & 21 & $(15,7)$ \\
Sangre & 10 & $(7,5)$ \\
Punta catéter & 3 & $(2,2)$ \\
Sec. Herida & & \\
Cultivo & 80 & $(59,7)$ \\
Negativo & 54 & $(40,3)$ \\
Positivo & & \\
\hline \hline
\end{tabular}

Se obtuvieron 54 aislamientos bacterianos de los cuales 28 $(51,8 \%)$ fueron bacterias productoras de BLEE (IC95\%: 38,5 a 65,2$)$. Las cuales se distribuyeron de la siguiente manera: $64,3 \%$ (IC95\%: 46,5 a 82,0) para E. coli y 35,7\% (IC95\%: 18,0 a 53,5 ) para $K$. pneumoniae (tabla 2). En cuanto a la producción de BLEE, esta fue investigada en las especies $K$. pneumoniae, E. coli y Proteus mirabilis (no hallado en el presente estudio), tal como lo sugiere el instituto de estándares clínicos y de laboratorio (CLSI). Asimismo, la distribución de frecuencias de las bacterias BLEE según grupo etario, edad y tipo de muestra se muestran en la tabla 3 .
Tabla 2. Distribución de frecuencias de las especies bacterianas productoras de BLEE más frecuentes en pacientes de la UCl del HDLM, Chiclayo. Enero a abril del 2018.

\begin{tabular}{lccccc}
\hline $\begin{array}{c}\text { Especie } \\
\text { bacteriana }\end{array}$ & N & (\%) & \multicolumn{2}{c}{$\begin{array}{c}\text { BLEE } \\
\text { BLEE/Total }(\%)\end{array}$} \\
\hline \hline E. coli & 19 & 35,19 & 18 & 64,29 & $18 / 19(94,7)$ \\
K. pneumoniae & 10 & 18,52 & 10 & 35,71 & $10 / 10(100,00)$ \\
A. baumani & 12 & 22,22 & 0 & 0,00 & $\ldots$ \\
P. aeuriginosa & 13 & 24,07 & 0 & 0,00 & $\ldots$ \\
Total & & & & & \\
& 54 & 100,00 & 28 & 100 & $28 / 54(51,9)$ \\
\hline
\end{tabular}

Tabla 3. Distribución de frecuencias de las bacterias BLEE según grupo etario, edad y tipo de muestra en pacientes de la UCI del HDLM, Chiclayo, enero a abril del 2018

\begin{tabular}{lcc}
\hline \multicolumn{1}{c}{ Variable } & BLEE/total & $(\%)$ \\
\hline Edad (años) & & \\
18 a 59 & $11 / 55$ & $(20,0)$ \\
60 a más & $15 / 68$ & $(22,1)$ \\
Menos de 18 & $2 / 11$ & $(18,2)$ \\
Sexo & & \\
Femenino & $13 / 60$ & $(21,7)$ \\
Masculino & $15 / 74$ & $(20,3)$ \\
Muestra & & \\
Orina & $10 / 25$ & $(40,0)$ \\
Punta catéter & $0 / 10$ & $(0,0)$ \\
Sangre & $3 / 21$ & $(14,3)$ \\
Sec. Bronquial & $14 / 75$ & $(18,7)$ \\
Sec. Herida & $1 / 3$ & $(33,3)$ \\
\hline
\end{tabular}

El estudio de sensibilidad y resistencia frente a los antimicrobianos, los aislamientos de enterobacterias productoras de BLEE presentaron un importante nivel de sensibilidad frente a carbapenemes (meropenem e imipenem), con un 96,4\% (IC95\%: 89,6 a 100,0), seguido de amikacina con un $82,1 \%$ (IC95\%: 67,9 a 96,3). En cuanto a sus niveles de resistencia, se presentó elevados niveles frente al resto de betalactámicos, debido a su naturaleza BLEE. Asimismo, se observó un elevado nivel de co-resistencia frente a amoxicilina/ácido clavulánico (100,0\%) y ciprofloxacino $(78,6 \%)$ (Ver figura 1$)$. 


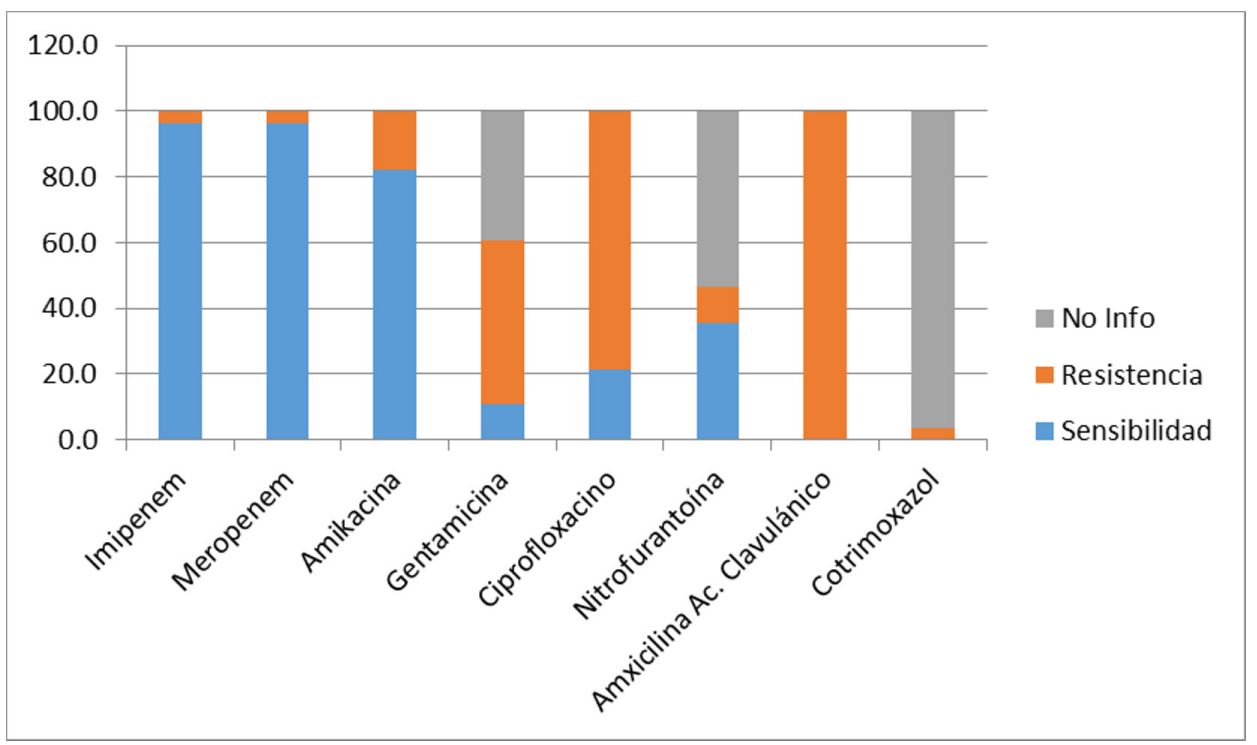

Figura 1. Distribución de frecuencias de la sensibilidad y resistencia antimicrobiana en enterobacterias productoras de BLEE aisladas en pacientes de la UCI del HRDLM, Chiclayo. Enero a abril del 2018.

\section{DISCUSIÓN}

La producción de BLEE es un factor asociado a mortalidad en bacteriemias causadas por enterobacterias en el ámbito hospitalario. Los pacientes infectados por estas cepas no solo tienen mayores tasas de mortalidad sino que desarrollan más complicaciones ${ }^{(10)}$.

Así mismo, en las áreas de cuidado crítico representan el principal grupo de microorganismos con multiresistencia (5). En nuestro estudio, de los 134 pacientes ingresados a áreas críticas del HDRLM durante el periodo enero a abril 2018, $54(40,3 \%)$ presentaron cultivos positivos, donde la E. coli productora de BLEE fue la especie que se halló con mayor frecuencia $(35,19 \%[n=19])$. Este resultado concuerda con otros estudios realizados en ámbito hospitalario donde reportaron $45,4 \%$ de E. coli ${ }^{(13)}$; así mismo, estudios nacionales permiten conocer que la frecuencia de esta bacteria en hospitales de Lima es muy similar $(55,8 \%)^{(14)}$. Es importante indicar que en los últimos años los nosocomios presentan una tendencia a incrementar en su frecuencia de $E$. coli, a comparación de K. pneumoniae, que se ha mantenido estable; esto revela el empoderamiento de esta especie frente a la adquisición de este fenotipo de resistencia ${ }^{(12,15,16)}$.

Por otro lado en la misma localidad donde se ejecutó el presente estudio se demostró que la colonización bacteriana por E. coli es muy frecuente en UCl ${ }^{(15)}$, lo cual sustentaría nuestro hallazgo, debido a que es conocido que toda infección es precedida por colonización. Aunque E. coli aparece como la principal enterobacteria en unidades críticas, un estudio multicéntrico realizado en $13 \mathrm{UCl}$ europeas, utilizando datos de vigilancia prospectiva y un modelo ma- temático para estimar las capacidades de transmisión y los números de reproducción de admisión única (RA ) de Escherichia coli y enterobacterias diferentes; observaron que $K$. pneumoniae era 3,7 veces más transmisibles que E. coli ${ }^{(17)}$. Este hallazgo explicaría por qué esta bacteria a pesar de presentar una elevada frecuencia en este tipo de servicios no representa el principal agente causal de brotes.

En cuanto a la sensibilidad antimicrobiana de las bacterias productoras de BLEE, en el presente estudio los carbapenemes y aminoglucósidos (con excepción de gentamicina) presentaron elevados niveles de sensibilidad, lo que los convierte en la mejor alternativa a emplear en este servicio. Dicho fenotipo de sensibilidad ha sido también observado en el estudio de Colquehuanca et al.(2015), quienes hallaron que las enterobacterias productoras de BLEE aisladas de coprocultivos en el Instituto Nacional de Salud del Niño, Lima-Perú presentaban un nivel de resistencia a la amikacina de $1,3 \%$, siendo todos sensibles al imipenem y meropenem ${ }^{(18)}$. De este modo, los niveles de resistencia a la amikacina se presentan muy bajos en estudios realizados en Perú, lo cual debería tomarse en cuenta como opción terapéutica para este tipo de aislamientos. De hecho, el uso excesivo de carbapenémicos podría limitar su uso en el futuro próximo ${ }^{(19)}$.

Anteriormente no se recomendaba el uso de aminoglucósidos por la frecuente corresistencia que se observaba en ellos, al igual que quinolonas. No obstante en los últimos años hemos aprendido que el tipo de BLEE y la epidemiologia de cada lugar juegan un rol importante, y en estudios 
recientes respecto al tema del tratamiento de BLEE se recomiendan el uso de aminoglucósidos en combinación con otros antimicrobianos, especialmente en infecciones urinarias e infecciones intrabdominales ${ }^{(20)}$. Así mismo, los aminoglucósidos administrados mediante nebulización alcanzan concentraciones muy elevadas en las vías respiratorias; y algunas observaciones sugieren que, en pacientes con neumonía asociada a la ventilación mecánica debido a microorganismos multirresistentes, pueden tener un efecto clínico beneficioso y prevenir el desarrollo ulterior de resistencia a los antibióticos administrados por vía intravenosa ${ }^{(21)}$.

Los carbapenemes representan una de las últimas líneas de defensa terapéutica que tenemos frente a bacilos gramnegativos y son el tratamiento de elección en aislamientos nosocomiales frente a bacterias productoras de BLEE. Por ello, el gran interés por preservarlos para situaciones especiales, como la estancia en cuidados crítico. Así mismo una de las estrategias que se realizan en el tratamiento de enfermedades infecciosas sobre todo a nivel nosocomial es el desescalonamiento terapéutico, que consiste en sustituir la terapia de amplio espectro por otra de menor espectro cuando se obtiene el resultado del estudio microbiológico (22). En este sentido, la sensibilidad que se presenta a amikacina $(82,1 \%)$ debería ser considerada para su empleo en combinación con otros antimicrobianos. Esto evitaría el uso de carbapenemes los cuales necesitan ser reservados para situaciones puntuales como en las infecciones producidas por $P$. aeruginosa y $A$. baumannii en donde ya están dejando de ser efectivos ${ }^{(23)}$.

En cuanto a la resistencia de enterobacteriáceas productoras de BLEE se resalta que en nuestro estudio hubo un completo nivel de resistencia frente a amoxicilina/ac. Clavulánico. de acuerdo a la definición de BLEE los inhibidores de betalactamasa deben de presentarse sensibles, la resistencia a estos inhibidores puede asociarse a otros mecanismos concomitantes como la producción de AmpC, oxacilinasas tipo 1 (OXA-1) o producción de IRT (inhibitor-resistant TEM mutant) (24).

En contraste con este hallazgo debemos indicar que la resistencia a inhibidores de la betalactamas varía con el tipo de BLEE y con el tipo de betalactamasa. Así por ejemplo, tazobactam es mejor que ácido clavulánico en presencia de CTX-M y ambos son mejores que sulbactam en presencia de TEM y SHV. Aunque existe poca evidencia clínica, algunos resultados favorables fueron encontrados en relación al uso de piperacilina/tazobactam. Sin embargo, estudios farmacodinámicos y farmacocinéticos muestran que piperacilina/tazobactam tiene menor actividad que cefepime. En un estudio realizado recientemente se encontró similares resultados en el tratamiento de bacteriemia por E. coli productora de BLEE con piperacilina/tazobactam, amoxicilina/ácido clavulánico y carbapenemes ${ }^{(25)}$.
Sin embargo debemos precisar que Amoxicilina/ácido clavulánico no parece ser una alternativa en el tratamiento de infecciones del tracto urinario adquiridas en la comunidad y mucho menos en cepas de origen hospitalario. Ante ello Ceftazidima/avibactam y Ceftolozano/tazobactam son los inhibidores de betalactamasa que en la actualidad representarian buenas opciones frente al tratamiento de BLEE. En este sentido Ceftazidima/avibactam ha sido estudiada en infecciones nosocomiales, específicamente neumonía adquirida en el hospital (NAH) y neumonía asociada al ventilador (NAV) y en estudios comparativos a meropenem no ha mostrado inferioridad, por su parte Ceftolozano/tazobactam aunque destinado para infecciones abdominales, ha demostrado adecuada exposición del tracto respiratorio inferior por su penetración al líquido del revestimiento intersticial, incluso mejor que piperacilina/tazobactam ${ }^{(8,21)}$.

La alta resistencia a las quinolonas observada en nuestro estudio $(78,6 \%)$, se correlaciona con lo observado por otros estudios a nivel hospitalarios donde se registran niveles de resistencia de hasta $91,7 \%{ }^{(26)}$. Esto indica que el uso de ciprofloxacino es completamente incompatible con el tratamiento de infecciones producidas por este tipo de bacterias. Este hecho se debe al uso tan extendido de este antimicrobiano para la mayoría de infecciones no solo de origen urinario sino intestinal y respiratorio. Lo cual, ha llevado a su agotamiento y a que en la actualidad su uso se restrinja a situaciones donde se halla evidencia microbiológica de su sensibilidad. Cabe mencionar que el acceso a éste y otros antibióticos sin prescripción médica en Perú es también un factor mayor, que contribuye al desarrollo de resistencia para lo cual será necesario implementar programas integrales de vigilancia y control epidemiológico, con la participación informada del profesional de salud.

Se concluye que las bacterias BLEE son frecuentes en aislamientos a partir de muestras de pacientes de la unidad de cuidados intensivos de un hospital público del norte del Perú. Se observó que la mayor frecuencia de aislamientos se obtiene de secreciones bronquiales; así mismo, la sensibilidad a los carbapenémicos y amikacina los propone como las mejores alternativas terapéuticas, aunque es sustancial preservarlos para infecciones por otros microorganismos multirresistentes. En este contexto es importante motivar estudios de efectividad frente a otros antimicrobianos que evite el agotamiento de los mismos y la presión selectiva. Por último, la alta resistencia al ciprofloxacino limita su uso en el ámbito hospitalario.

Fuentes de financiamiento. Autofinanciado.

Conflicto de interés. Los autores no poseen conflicto de interés. 


\section{REFERENCIAS BIBLIOGRÁFICAS}

1. Laudy AE, Róg P, Smolińska-Król K, Ćmiel M, Słoczyńska A, Patzer J, et al. Prevalence of ESBL-producing Pseudomonas aeruginosa isolates in Warsaw, Poland, detected by various phenotypic and genotypic methods. Gonzalez-Bello C, editor. PLoS One. 2017;12(6):e0180121.

2. Safari M, Mozaffari Nejad AS, Bahador A, Jafari R, Alikhani MY. Prevalence of ESBL and MBL encoding genes in Acinetobacter baumannii strains isolated from patients of intensive care units (ICU). Saudi J Biol Sci. 2015; 22(4):424-9.

3. Tejada-Llacsa PJ, Huarcaya JM, Melgarejo GC, Gonzales LF, Cahuana J, Pari RM, et al. Caracterización de infecciones por bacterias productoras de BLEE en un hospital de referencia nacional. An la Fac Med. 2015; 76(2):161-6.

4. Carrillo B. Bacterias Productoras de BLEE. Botica. 2014 [cited 2018 Jun 24]. p. 3. Available from: http://botica.com.ve/PDF/bleeB21.pdf

5. Barrera Monterroso ML. Determinación del perfil de resistencia antibiótica de Escherichia coli, Klebsiella oxytoca y Klebsiella pneumoniae en el Sanatorio privado "Nuestra Señora de Pilar". Tesis; Universidad de San Carlos de Guatemala; 2005. Available from: http://biblioteca.usac.edu.gt/ tesis/06/06_2329.pdf

6. Morones-Esquivel I, Salgado-Muñoz T, Gonzaga-López T, Matamoros-Mejía A, Terán-González J, Arteaga-Vázquez S, et al. Enterobacterias con betlactamasas de espectro extendido en hemocultivos y urocultivos. Med Int Mex. 2016; 32(4):381-7.

7. Kengne M, Dounia AT, Nwobegahay JM. Bacteriological profile and antimicrobial susceptibility patterns of urine culture isolates from patients in Ndjamena, Chad. Pan Afr Med J. 2017 [cited 2018 Jun 24];28:258. Disponoble en: http://www.ncbi.nlm.nih.gov/pubmed/29881501

8. Olarte-Luis T, Cáceres-Galíndez D, Cortés JA. Nuevas cefalosporinas. Rev Chil Infectol. 2018; 35(5):465-75.

9. Penadillo Huashuayo ML, Rosas Carhuayal MV, Benites Azabache JC. Carcaterización fenotípica de las bacterias causantes de infecciones intrahospitalarias en pacientes del Hospital Nacional Arzobispo Loayza, durante el 2016. Tesis: Universidad Norbert Wiener; 2017. Disponible en: http://repositorio.uwiener.edu.pe/bitstream/handle/123456789/1528/ TITULO - Rosas Carhuayal\%2C Malena Virginia.pdf?seque

10. Adrianzén D, Arbizu Á, Ortiz J, Samalvides F. Mortalidad por bacteriemia causada por Escherichia coli y Klebsiella spp. productoras de beta lactamasas de espectro extendido: cohorte retrospectiva en un hospital de Lima, Perú. Rev Peru Med Exp Salud Publica. 2013; 30(1):18-25.

11. Mendoza Hernández EA, Ocaña Paredes CA, Salazar Zuloeta J, Pecsén Monteza JE. Factores de riesgo para infección de tracto urinario por gérmenes productores de betalactamasas de espectro extendido en el servicio de medicina de Hospital Provincial Docente Belén de Lambayeque, durante el año 2016. Tesis: Universidad Nacional Pedro Ruiz Gallo; 2017. Disponible en: http://repositorio.unprg.edu.pe/bitstream/handle/ UNPRG/924/BC-TES-5709.pdf? sequence=1\&isAllowed=y

12. Bustamante Canelo $\mathrm{O}$. Escherichia coli y Klebsiella pneumoniae betalactamasa de espectro extendido en pacientes con infección intrahospitalaria del tracto urinario, Hospital Regional Lambayeque. enero - julio 2015. Tesis: Universidad Nacional Pedro Ruiz Gallo; 2017. Disponible en: http://repositorio.unprg.edu.pe/bitstream/handle/UNPRG/1256/BCTES-TMP-88.pdf?sequence=1\&isAllowed=y

13. Sobhan Ghafourian al, Sadeghifard N, Soheili S, Sekawi Z. Extended Spectrum Beta-lactamases. Curr Issues Mol Biol. 2015; 17:11-22.

14. Espinoza C, Acosta Acosta LS, Escobar S. Determinar la resistencia antimicrobiana de las enterobacterias y el uso de antibióticos en pacientes de UCl de la Clínica D.A.M.E. 2014.Tesis: Escuela Superior Politécnica de Chimborazo; 2015. Disponible en: http://dspace.espoch.edu.ec/handle/123456789/3890

15. Aguilar Gamboa FR, Aguilar Martinez SL, Cubas Alarcón DM, Coaguila Cusicanqui LÁ, Fernández Valverde DA, Mario Cecilio MM, et al. Portadores de bacterias multirresistentes de importancia clínica en áreas críticas (UCI-UCIN) de un hospital al norte del Perú. Horiz Med. 2016; 16(3):50-7.

16. Shakya P, Shrestha D, Maharjan E, Sharma VK, Paudyal R. ESBL Production Among E. coli and Klebsiella spp. Causing Urinary Tract Infection: A Hospital Based Study. Open Microbiol J. 2017; 11:23-30.

17. Pierce KE, Peter H, Bachmann TT, Volpe C, Mistry R, Rice JE, et al. Rapid Detection of TEM-Type Extended-Spectrum $\beta$-Lactamase (ESBL) Mutations Using Lights-On/Lights-Off Probes with Single-Stranded DNA Amplification. J Mol Diagnostics. 2013; 15(3):291-8.
18. Colquehuanca Aliaga F, Sevilla Andrade C, Gonzales Escalante E. Enterobacterias productoras de betalactamasas de espectro extendido en muestras fecales en el Instituto Nacional de Salud del Niño, Perú. Rev Peru Med Exp Salud Publica. 2002; 32(1):26-32.

19. Zahedi Bialvaei A, Samadi Kafil H, Ebrahimzadeh Leylabadlo H, Asgharzadeh M, Aghazadeh M. Dissemination of carbapenemases producing Gram negative bacteria in the Middle East. Iran J Microbiol. 2015; 7(5):226-46.

20. Pana ZD, Zaoutis T. Treatment of extended-spectrum $\beta$-lactamase-producing Enterobacteriaceae (ESBLs) infections: what have we learned until now? F1000Research. 2018; 7(PMC6117850).

21. Martínez JA. Approach to directed therapy after knowledge of the isolate: carbapenemase-producing Enterobacteriaceae, multidrug-resistant Pseudomonas aeruginosa and carbapenem-resistant Acinetobacter baumannii. Rev Esp Quim. 2016; 29(Suppl. 1):31-4.

22. Berenguer Torrijo N, Grau Cerrato S, Saballs Radresa P, Sáez-Benito L. Desescalada terapéutica en pacientes que ingresan en urgencias con infecciones adquiridas en el ámbito extrahospitalario tratados de inicio con imipenem. Tesis: Universidad Autónoma de Barcelona; 2015. Disponible en: https://www.tesisenred.net/bitstream/handle/10803/316574/ nbt1de1.pdf?sequence=1\&isAllowed $=y$

23. Akhi MT, Khalili Y, Ghottaslou R, Aghazadeh M, Seroush Bar Hagh MHI, Yousefi S. Prevalence of PER-1- type Extended-Spectrum Beta-Lactamaes in Clinical Strains of Pseudomonas aeruginosa Isolated from Tabriz, Iran. Iran J Basic Med Sci. 2012; 15(1):678-82.

24. Oteo J, González-López JJ, Ortega A, Quintero-Zárate JN, Bou G, Cercenado E, et al. Inhibitor-Resistant TEM- and OXA-1-Producing Escherichia coli Isolates Resistant to Amoxicillin-Clavulanate Are More Clonal and Possess Lower Virulence Gene Content than Susceptible Clinical Isolates. Antimicrob Agents Chemother. 2014; 58(7):3874-81.

25. Lezameta L, Gonzáles-Escalante E, Tamariz JH. Comparación de cuatro métodos fenotípicos para la detección de beta-lactamasas de espectro extendido. Rev Peru Med Exp Salud Publica. $2010 ; 27(3): 345-51$.

26. Thaden JT, Fowler VG, Sexton DJ, Anderson DJ, Anderson DJ. Increasing Incidence of Extended-Spectrum $\beta$-Lactamase-Producing Escherichia coli in Community Hospitals throughout the Southeastern United States. Infect Control Hosp Epidemiol. 2016; 37(1):49-54

Revisión de pares: Recibido: 06/07/2019 Aceptado: 25/07/2019 